\title{
Surface display of designer protein scaffolds on genome-reduced
}

\section{strains of Pseudomonas putida}

by

Pavel Dvořák ${ }^{1^{*}}$, Edward A. Bayer ${ }^{2}$, and Víctor de Lorenzo ${ }^{3 *}$

${ }^{1}$ Department of Experimental Biology (Section of Microbiology), Faculty of Science, Masaryk University, Kamenice 753/5, 62500, Brno, Czech Republic.

${ }^{2}$ Department of Biomolecular Sciences, The Weizmann Institute of Science, Rehovot 76100, Israel

${ }^{3}$ Systems and Synthetic Biology Program, Centro Nacional de Biotecnología CNB-CSIC, Cantoblanco, Darwin 3, 28049 Madrid, Spain.

Keywords: Pseudomonas putida, cellulosome, designer scaffoldin, surface display, synthetic biology

* Co-corresponding authors:

Prof. V. de Lorenzo

Systems and Synthetic Biology Program, Centro Nacional de Biotecnología (CNB-CSIC)

Darwin 3, Campus de Cantoblanco Madrid 28049, Spain

Phone: +34 91585 4536, Fax: +34 91585 4506, E-mail: vdlorenzo@cnb.csic.es

Dr. Pavel Dvořák

Department of Experimental Biology (Section of Microbiology), Faculty of Science

Masaryk University, Kamenice 735/5, Brno 62500, Czech Republic

Phone: +420 549493 396, E-mail: pdvorak@sci.muni.cz 
Supplementary methods .3

Supplementary tables .5

Table S1. Strains and plasmids used in this study.

Table S2. Oligonucleotide primers used in this study.

Supplementary figures 9

Figure S1. Schematic reconstruction of genomic deletions in Pseudomonas putida strains EM42 and EM371 used in this study.

Figure S2. Effect of codon optimization of the scaf19L gene on toxicity, solubility, and stability of the corresponding protein produced in Pseudomonas putida EM42.

Figure S3. SDS-polyacrylamide gels with samples from purification of BglC, BglC-CtDoc, Bglc-AcDoc, GFP-CtDoc, and CFP-AcDoc.

Figure S4. Effect of an autotransporter expression on viability of the host bacterium Pseudomonas putida EM42.

Figure S5. Expression and display analysis of three different autotransporters with CtCoh passenger in Pseudomonas putida EM42 and EM371.

Figure S6. Expression analysis of three miniscaffoldin variants displayed via Ag43 autotransporter in Pseudomonas putida EM42 and EM371.

Supplementary sequences

References. 


\section{Supplementary methods}

Effect of scaffoldin and autotransporter expression on the viability of P. putida cells. Overnight cultures of $P$. putida EM42 cells bearing pSEVA238b_scaf19L, pSEVA238_scaf19LKT, or empty pSEVA238 were used to inoculate $20 \mathrm{~mL}$ of LB with $\mathrm{Km}$ in $100 \mathrm{~mL}$ shake flasks to initial $\mathrm{OD}_{600}$ of 0.05 . The cells were grown for three hours with shaking (170 rpm) and then $0.5 \mathrm{mM} 3$-methylbenzoate (3MB) was added to all cultures to induce expression of recombinant genes. The cells were further cultured for $18 \mathrm{~h}$, and $\mathrm{OD}_{600}$ was measured periodically. The effect of IgAAT, IntAT, Ag43AT, and EstPAT autotransporter expression from the pSEVA238 plasmid on viability of the EM42 host was tested correspondingly.

SDS-PAGE and western blot analyses. Cell-free extracts (CFEs) and purified proteins for the evaluation of purification procedure were prepared as described in the Materials and Methods section Protein purification by affinity chromatography in the main text. Cell lysates for the evaluation of expression levels of recombinant proteins in P. putida EM42 and EM371 were prepared as follows. Cells, collected from $10 \mathrm{~mL}$ of LB medium culture and washed with TBS buffer, were added with $250 \mathrm{uL}$ of B-PER Bacterial Protein Extraction Reagent, $0.25 \mu \mathrm{L}$ of lysozyme and $0.25 \mu \mathrm{L}$ of DNase I (all components from Thermo Fisher Scientific, USA) and lysed for $15 \mathrm{~min}$ at room temperature with slow agitation. For separation of soluble and insoluble fractions (see Effect of scaffoldin and autotransporter expression on the viability of P. putida cells in Materials and Methods section in the main text), cell lysates were centrifuged $\left(21,000 \mathrm{~g}, 4^{\circ} \mathrm{C}, 30 \mathrm{~min}\right)$, supernatants (CFEs) were collected and pellets (insoluble fractions) were washed with distilled water, centrifuged and re-suspended in $50 \mu \mathrm{L}$ of distilled water. Samples of CFE, as well as samples of wash, elution, or insoluble fractions or purified chimeric proteins, were added with $5 \mathrm{x}$ Laemmli buffer, boiled at $100{ }^{\circ} \mathrm{C}$ for $7 \mathrm{~min}$ and separated by SDS-PAGE using $12 \%$ gels. In all cases, $5 \mu$ g of total protein were loaded per gel well. Gels were stained with Coomassie Brilliant Blue R-250 (Fluka/Sigma-Aldrich, Switzerland). Protein purity or quantity in cell lysates was estimated by using ImageJ $1.50 \mathrm{~b}$ or GS-800 Calibrated Densitometer with Quantity One 1-D software v4.6 (Bio-Rad, USA). 
The staining step was omitted for western blot analyses. Samples of CFE or insoluble fractions ( $5 \mu \mathrm{g}$ of total protein per well) were loaded on $12 \%$ polyacrylamide gel. After SDS-PAGE, proteins were electrotransferred during a 30-min interval from the gel onto an Immobilon-P membrane of pore size of $0.45 \mu \mathrm{m}$ (Merck Millipore, USA) under constant electric current of 0.1 A per gel and voltage of 5-7 V using Trans-Blot SD Semi-Dry Transfer Cell (Bio-Rad, USA). Alternatively, wet transfer was conducted under constant current of 0.375 A per gel using Mini-PROTEAN Tetra Vertical Electrophoresis Cell (Bio-Rad, USA) during a 120-min interval. The gels were stained with Coomassie Brilliant Blue R-250 (Fluka/Sigma-Aldrich, Switzerland) to confirm complete protein transfer. The membrane was blocked overnight at $4^{\circ} \mathrm{C}$ in PBS buffer with $3 \%(\mathrm{w} / \mathrm{v})$ dry milk and $0.1 \%(\mathrm{v} / \mathrm{v})$ Tween 20 and then incubated with mouse anti-6xHis tag monoclonal antibody-HRP conjugate (Clontech or Thermo Fisher Scientific, USA) in the same buffer for $2 \mathrm{~h}$ at room temperature. PBS buffer with $1 \%(\mathrm{v} / \mathrm{v})$ Tween 20 was employed for membrane washing (4-times $5 \mathrm{~min}$ ), and the proteins were then visualized with SuperSignal West Pico PLUS Chemiluminescent Substrate (Thermo Fisher Scientific, USA) using FUSION Solo S documentation system (Vilber, Germany).

Dot blot analysis. P. putida recombinants were cultured as described in chapter Evaluation of scaffoldin display and cohesin-dockerin binding on P. putida cell surface by $\beta$-glucosidase activity assay in Materials and Methods section of the main text. Cells from $10 \mathrm{~mL}$ of LB medium culture were collected by centrifugation $\left(2370 \mathrm{~g}, 4^{\circ} \mathrm{C}, 5 \mathrm{~min}\right)$, washed twice with icecold TBS buffer with $0.05 \%$ Tween 20 (TBS-T) and resuspended in $1 \mathrm{~mL}$ of the same buffer to the final $\mathrm{OD}_{600}$ of 5.0. Cells were dotted $(2 \mu \mathrm{L}$ per dot $)$ on the nitrocellulose membrane of pore size of $0.45 \mu \mathrm{m}$ (Thermo Fisher Scientific, USA), and the membrane was dried at $30{ }^{\circ} \mathrm{C}$ for 15 min. The membrane was blocked in TBS buffer with $3 \%(\mathrm{w} / \mathrm{v})$ dry milk and $0.05 \%$ (v/v) Tween 20 for $30 \mathrm{~min}$ at room temperature and then incubated with mouse anti-6xHis tag monoclonal antibody-HRP conjugate (Thermo Fisher Scientific, USA) in TBS-T with $0.05 \%$ BSA for $2 \mathrm{~h}$ at room temperature. The membrane was washed 3 times for 5 min with TBS-T and once with TBS, and the His-tagged proteins on the cell surface were then visualized with SuperSignal West Pico PLUS Chemiluminescent Substrate (Thermo Fisher Scientific, USA) using FUSION Solo S documentation system (Vilber, Germany). 
Supplementary Table S1. Strains and plasmids used in this study.

\begin{tabular}{|c|c|c|}
\hline Strain or plasmid & Characteristics & $\begin{array}{l}\text { Source or } \\
\text { reference }\end{array}$ \\
\hline \multicolumn{3}{|l|}{ Escherichia coli } \\
\hline DH5 $\alpha$ & 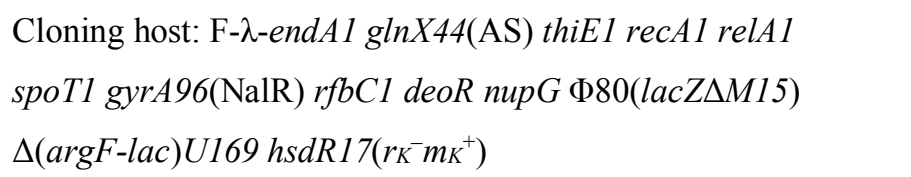 & Grant et al. ${ }^{1}$ \\
\hline CC118 & $\begin{array}{l}\text { Cloning host: } \Delta \text { (ara-leu) araD } \triangle \text { lac X174 galE galK phoA } \\
\text { thiE1 rpoB }\left(\text { Rif }^{R}\right) \arg E(A m) \text { recAl }\end{array}$ & $\begin{array}{l}\text { Manoil and } \\
\text { Beckwith }^{2}\end{array}$ \\
\hline BL21-Gold (DE3) & $\begin{array}{l}\text { Expression host, E. coli B derivative: } \mathrm{F}^{-} \text {omp } T \text { hsdS }\left(\mathrm{r}^{-} \mathrm{m}_{\mathrm{B}}^{-}\right) \\
d c m^{+} \text {Tet }^{\mathrm{r}} \text { gal } \lambda\left(\mathrm{DE} 3\left[\text { lacl }^{Q} \text { lacUV5-T7 gene } 1 \text { ind1 } \operatorname{sam} 7 \text { nin } 5\right]\right) \\
\text { endA } \mathrm{Hte}\end{array}$ & $\begin{array}{l}\text { Agilent } \\
\text { Technologies, } \\
\text { USA }\end{array}$ \\
\hline HB101 & $\begin{array}{l}\text { Helper strain for tri-parental mating: } \mathrm{F}-\lambda-h s d S 20\left(\mathrm{r}^{-} \mathrm{mB}^{-}\right) \\
\text {recA13 leuB6(Am) araC14 } \Delta(\text { gpt-proA }) 62 \text { lacY1 galK2(OC) } \\
x y l-5 \text { mtl-1 thiE1 rpsL20 }\left(\mathrm{Sm}^{\mathrm{R}}\right) \operatorname{gln} X 44(\mathrm{AS})\end{array}$ & $\begin{array}{l}\text { Boyer and } \\
\text { Roulland- } \\
\text { Dussoix }{ }^{3}\end{array}$ \\
\hline \multicolumn{3}{|c|}{ Pseudomonas putida } \\
\hline EM42 & $\begin{array}{l}\text { Derivative of strain P. putida KT2440: } \Delta \text { prophages 1,2,3,4 } \\
\Delta \operatorname{Tn} 7 \Delta \text { endA1 } \triangle \text { endA2 } \triangle h s d R M S \Delta \text { flagellum } \Delta \operatorname{Tn} 4652\end{array}$ & $\begin{array}{l}\text { Martínez-García } \\
\text { et al. }^{4}\end{array}$ \\
\hline EM371 & $\begin{array}{l}\text { Derivative of strain } P \text {. putida KT2440: } \Delta \text { prophage } 4 \Delta \operatorname{Tn} 7 \\
\Delta \text { flagellum } \Delta \text { pili } \Delta \text { curli } \Delta \text { motility proteins } \Delta \text { alginate } \\
\text { biosynthesis } \Delta \text { twitching motility protein } \Delta \text { surface adhesion } \\
\text { protein } \Delta \text { cellulose synthesis } \Delta \text { outer membrane lipoprotein } \\
\Delta \text { glycosyl transferase }\end{array}$ & $\begin{array}{l}\text { Martínez-García } \\
\text { et al. }\end{array}$ \\
\hline \multicolumn{3}{|l|}{ plasmids } \\
\hline pRK600 & $\begin{array}{l}\text { Helper plasmid for tri-parental mating: oriV(ColE1) RK2 } t^{+} a^{+} \\
\mathrm{mob}^{+}, \mathrm{Cm}^{\mathrm{R}}\end{array}$ & Kessler et al. ${ }^{6}$ \\
\hline pSEVA238 & Expression vector: oriV(pBBR1) xylS-Pm neo, $\mathrm{Km}^{\mathrm{R}}$ & $\begin{array}{l}\text { Silva-Rocha et } \\
\text { al. }{ }^{7}\end{array}$ \\
\hline pSEVA238b & $\begin{array}{l}\text { Derivative of pSEVA238 with synthetic RBS and adjacent } \\
N d e I \text { site for subcloning of genes to be expressed }\end{array}$ & $\begin{array}{l}\text { Dvořák and de } \\
\text { Lorenzo }^{8}\end{array}$ \\
\hline pSEVA238_gfp & $\begin{array}{l}\text { pSEVA238 bearing synthetic RBS and gene of monomeric } \\
\text { superfolder GFP (msfGFP) cloned into HindIII and SpeI sites } \\
\text { of SEVA polylinker }\end{array}$ & SEVA collection \\
\hline pET21b & $\begin{array}{l}\text { Expression vector: ori }(\mathrm{pBR} 322, \mathrm{f1}), \mathrm{T} 7 \text { promoter and } \\
\text { terminator, } l a c I, \mathrm{Amp}^{\mathrm{R}}\end{array}$ & Merck, USA \\
\hline pET28a_scaf19L & $\begin{array}{l}\text { Expression vector: ori }(\mathrm{pBR} 322, \mathrm{f} 1), \mathrm{T} 7 \text { promoter and } \\
\text { terminator, } l a c I, \mathrm{Km}^{\mathrm{R}} \text {, with synthetic scaffoldin gene } s c a f 19 \mathrm{~L}\end{array}$ & Vazana et al. ${ }^{9}$ \\
\hline
\end{tabular}


(encodes CBM3a and CohCt A2 of CipA from Clostridium

thermocellum, CohAc C3 of ScaC from Acetivibrio

cellulolyticus, CohBc B3 of ScaB from Bacteroides

cellulosolvens with 27-35 aa long linkers, and C-terminal

6xHis tag) cloned into NcoI and XhoI sites

pSEVA238b_scaf19L

pSEVA238b with scaffoldin gene scaf19L cloned into NdeI

This study and PstI sites

pUC57_scaf19LKT Cloning vector $\left(\mathrm{Amp}^{\mathrm{R}}\right)$ with scaf19L gene synthesized with

This study RBS and codon-optimized for expression in P. putida KT2440

pSEVA238_scaf19LKT pSEVA238 with synthesized scaf19L gene (with RBS) cloned

This study into SacI and KpnI sites

pUC57_ctDoc

pUC57_acDoc

pSEVA238b_bglC

pSEVA238b_bglC-

ctDoc

pSEVA238b_bglC-

acDoc

pSEVA238_gfpN

pSEVA238_gfp-ctDoc

pET21b_gfp-ctDoc

pET28a_cfp-acDoc

pUC57_estPAT

pAg43pol
Cloning vector $\left(A m p^{\mathrm{R}}\right)$ with synthetic ctDoc sequence (encodes C. thermocellum dockerin with C-terminal 6xHis tag) codon-optimized for expression in P. putida KT2440

Cloning vector $\left(\mathrm{Amp}^{\mathrm{R}}\right)$ with synthetic acDoc sequence (encodes $A$. cellulolyticus dockerin with C-terminal 6xHis tag) codon-optimized for expression in P. putida KT2440

pSEVA238b harboring $b g l C$ gene of $\beta$-glucosidase from

Thermobifida fusca with N-terminal 6xHis tag cloned into

NdeI and HindIII sites

pSEVA238b harboring chimeric bglC-ctDoc gene cloned into

NdeI and HindIII sites

pSEVA238b harboring chimeric $b g l C$-acDoc gene cloned into

NdeI and HindIII sites

pSEVA238 with $g f p$ gene encoding monomeric superfolder

green fluorescent protein (msfGFP) with synthetic RBS but

lacking STOP codon (AvrII/EcoRI)

pSEVA238_gfpN with $c t D o c$ gene cloned in frame

downstream the $g f p$ gene (SacI/HindIII) to form a translational fusion

pET21b with $g f p N-c t D o c$ cloned into NdeI and HindIII sites.

pET28a with $c f p$-acDoc gene (encodes cyan fluorescent protein

mCerulean fused to $A$. cellulolyticus ScaB dockerin) cloned into $X b a \mathrm{I}$ and $X h o I$ sites

This study

(GeneCust,

France)

This study

(GeneCust,

France)

Dvořák and de

Lorenzo $^{8}$

This study

This study

This study

This study

This study

Gift from

Edward A.

Bayer

This study

(GeneCust,

France)

Muñoz-

Gutiérrez et al. ${ }^{10}$ 


\begin{tabular}{|c|c|c|}
\hline pSEVA238_igAAT & $\begin{array}{l}\text { pSEVA238 bearing synthetic RBS and gene encoding C- } \\
\text { terminal part ( } 409 \text { AA) of IgA-specific serine endopeptidase } \\
\text { autotransporter from Neisseria gonorrhoeae with } 22 \text { AA N- } \\
\text { terminal PelB leader sequence of pectate lyase B of Erwinia } \\
\text { carotovora CE and SEVA polylinker, whole gene is cloned } \\
\text { into AvrII and SpeI sites }\end{array}$ & $\begin{array}{l}\text { Esteban } \\
\text { Martínez-García }\end{array}$ \\
\hline pSEVA238_intAT & $\begin{array}{l}\text { pSEVA238 bearing synthetic RBS and gene encoding N- } \\
\text { terminal part ( } 654 \text { AA) of intimin } \gamma \text { from enterohemorrhagic } E \text {. } \\
\text { coli O157:H7 with original } 39 \text { AA leader sequence, SEVA } \\
\text { polylinker is at 3' terminus, whole gene is cloned into AvrII } \\
\text { and SpeI sites }\end{array}$ & $\begin{array}{l}\text { Esteban } \\
\text { Martínez-García }\end{array}$ \\
\hline pSEVA238b_ag43AT & $\begin{array}{l}\text { pSEVA238b with gene encoding C-terminal part ( } 487 \mathrm{AA} \text { ) of } \\
\text { adhesin Ag43 autotransporter from E.col with original } 52 \mathrm{AA} \\
\text { leader sequence and inserted polylinker, whole gene is cloned } \\
\text { into NdeI and HindIII sites }\end{array}$ & This study \\
\hline pSEVA238b_estPAT & $\begin{array}{l}\text { pSEVA238b with gene encoding estPAT autotransporter } \\
\text { cloned into NdeI and HindIII sites }\end{array}$ & This study \\
\hline $\begin{array}{l}\text { pSEVA238_igAAT- } \\
\text { ctCoh }\end{array}$ & $\begin{array}{l}\text { pSEVA238_igAAT with gene encoding ctCoh cohesin codon- } \\
\text { optimized for expression in } P \text {. putida KT } 2440 \text { and cloned into } \\
\text { EcoRI and BamHI sites of igAAT polylinker }\end{array}$ & This study \\
\hline $\begin{array}{l}\text { pSEVA238_intAT- } \\
\text { ctCoh }\end{array}$ & $\begin{array}{l}\text { pSEVA238_intAT with gene encoding ctCoh cohesin codon- } \\
\text { optimized for expression in } P \text {. putida KT } 2440 \text { and cloned into } \\
\text { EcoRI and BamHI sites of intAT polylinker }\end{array}$ & This study \\
\hline $\begin{array}{l}\text { pSEVA238b_ag43AT- } \\
\text { ctCoh }\end{array}$ & $\begin{array}{l}\text { pSEVA238b_ag43AT with gene encoding ctCoh cohesin } \\
\text { codon-optimized for expression in P. putida KT2440 and } \\
\text { cloned into XhoI and BamHI sites of Ag43AT polylinker }\end{array}$ & This study \\
\hline $\begin{array}{l}\text { pSEVA238b_ag43AT- } \\
\text { acCoh }\end{array}$ & $\begin{array}{l}\text { pSEVA238b_ag43AT with gene encoding acCoh cohesin } \\
\text { codon-optimized for expression in P. putida KT2440 and } \\
\text { cloned into XhoI and BamHI sites of } A g 43 A T \text { polylinker }\end{array}$ & This study \\
\hline $\begin{array}{l}\text { pSEVA238b_ag43AT- } \\
\text { acCoh-ctCoh }\end{array}$ & $\begin{array}{l}\text { pSEVA238b_ag43AT with gene encoding acCoh-ctCoh } \\
\text { scaffoldin codon-optimized for expression in } P \text {. putida } \\
\text { KT2440 and cloned into XhoI and BamHI sites of } A g 43 A T \\
\text { polylinker }\end{array}$ & This study \\
\hline
\end{tabular}

Abbreviations: RBS, ribosome binding site; CBM, carbohydrate binding module; Cm, chloramphenicol; Amp, ampicillin; Km, kanamycin; Sm, streptomycin; Sp, spektinomycin; Coh, cohesin; Doc, dockerin. 
Supplementary Table S2. Oligonucleotide primers used in this study.

\begin{tabular}{|c|c|}
\hline Name & Sequence $\left(5^{\prime} \rightarrow 3^{\prime}\right)^{a}$ \\
\hline Sca19L fw (Ndel) & taacatatggcaaatacaccggtatcag \\
\hline Sca19L rv (Pstl) & aatctgcagtcagtggtggtggtggtg \\
\hline BglC-CtDoc TS1F (Ndel) & atacatatgcaccatcaccatcac \\
\hline BglC-CtDoc TS1R & ggaaccacccccggattcctgtccgaagattccc \\
\hline BglC-CtDoc TS2F & tccgggggtggttcc \\
\hline BglC-CtDoc TS2R (HindIII) & attaagcttttagtggtggtgatggtggtggttcttgtacggcagcgtgtc \\
\hline BglC-AcDoc TS1R & cacgtcgccgtagatgaacttgctagcggaaccacccc \\
\hline BglC-AcDoc TS2F & aagttcatctacggcgacgtg \\
\hline BglC-AcDoc TS2R (HindIII) & taaaagcttttagtgatggtgatggtggtg \\
\hline CtCoh fw (EcoRI, Xhol) & atagaattcagtcgacctcgagtccgatggcgtagtagtgg \\
\hline CtCoh rv1 (BamHI) & tatctgcagggatccgtgatggtggtgatggtg ggtggcgttgccc \\
\hline CtCoh rv2 (BamHI) & tatggatccatgtgatggtggtgatggtgggtggcgttgccc \\
\hline AcCoh fw (Xhol) & atactcgagggttccgatttgcaggtggac \\
\hline AcCoh rv (BamHI) & tatggatccgtgatggtggtgatggtggctggcgatcacctcgatc \\
\hline AcCoh-CtCoh fw (Xhol) & atagaattcagtcgacctcgagggttccgatttgcaggtg \\
\hline GFP-N fw (Avrll) & attcctaggtagcaagaggaatatcatatgagtaaaggagaagaacttttcac \\
\hline GFP-N rv (EcoRI) & aatgaattctttgtatagttcatccatgcc \\
\hline CtDoc fw (Sacl) & aatgagctctccgggggtggttcc \\
\hline CtDoc rv (HindIII) & cgcaagcttttagtggtggtg \\
\hline
\end{tabular}

${ }^{a}$ Restriction sites are underlined. 


\section{Supplementary Figures}

Supplementary Figure S1. Schematic reconstruction of genomic deletions in Pseudomonas putida strains EM42 and EM371 used in this study

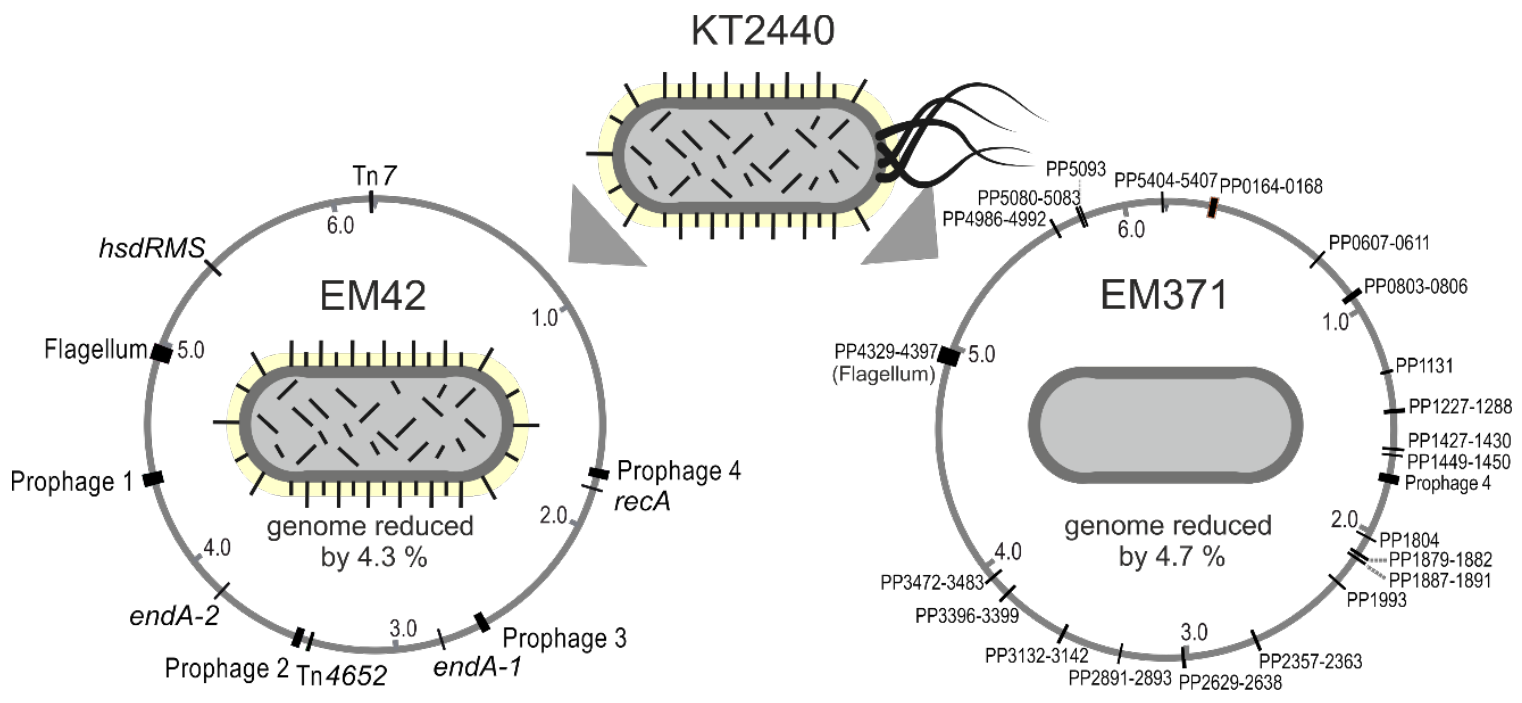

For more details on P. putida EM42 and EM371 see original studies by Martínez-García et al. ${ }^{4}$ and Martínez-García et al. ${ }^{5}$, respectively. 
Supplementary Figure S2. Effect of codon optimization of the scaf19L gene on toxicity, solubility, and stability of the corresponding protein produced in Pseudomonas putida EM42.

(a)

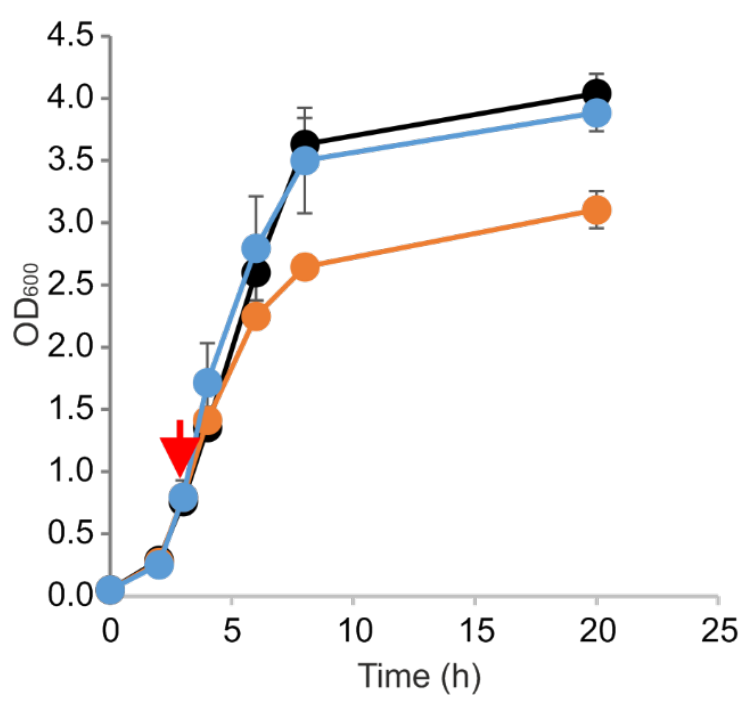

(b)

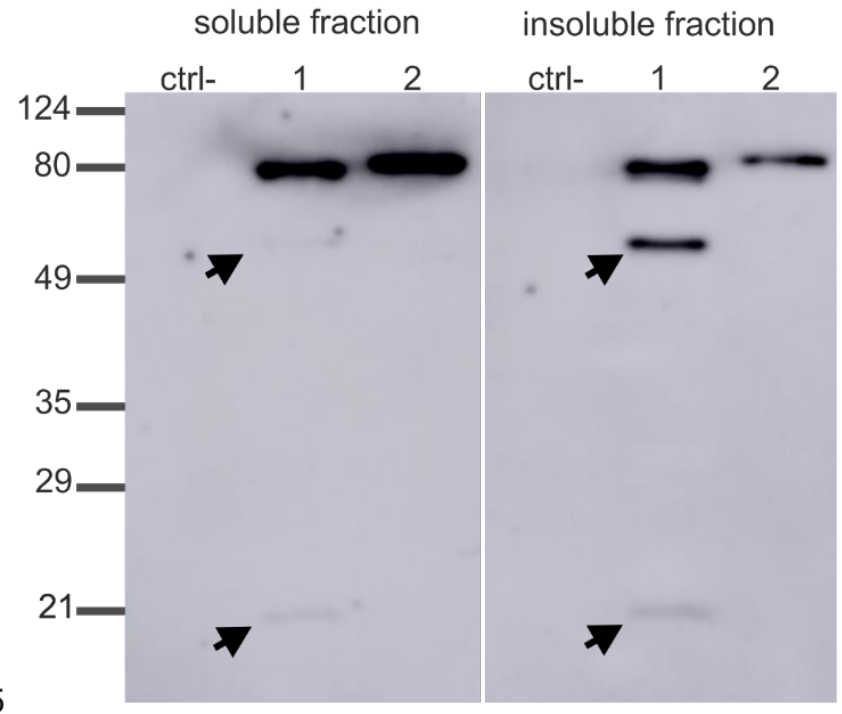

(a) Effect of scaf19L (orange line) and scaf19LKT (blue line) expression on viability of $P$. putida EM42 host. Cells were cultured in $20 \mathrm{~mL}$ of lysogeny broth at $30^{\circ} \mathrm{C}$ with shaking $(170$ $\mathrm{rpm})$. Expression of the heterologous gene cloned in pSEVA238 plasmid was induced $3 \mathrm{~h}$ after culture start (red arrow) with $0.5 \mathrm{mM} 3$-methylbenzoate. P. putida EM42 with empty pSEVA238 plasmid (black line) was used as a control. Data are shown as mean \pm SD from two biological replicates. (b) Western blot analysis of Scaf19L (1) and Scaf19LKT (2) with Cterminal 6xHis tag in soluble (cell-free extract) and insoluble fraction of cell lysate. Protein of the theoretical molecular weight of $75.4 \mathrm{kDa}$ was detected using mouse anti-6xHis tag monoclonal antibody-HRP conjugate (Clontech, USA). Black arrows indicate possible products of Scaf19L proteolysis. Protein marker is in $\mathrm{kDa}$. 
Supplementary Figure S3. SDS-polyacrylamide gels with samples from purification of five used recombinant proteins.

(a)

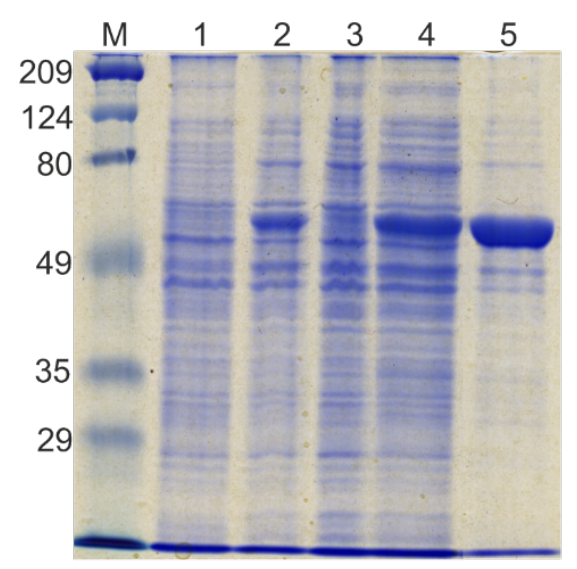

(d)

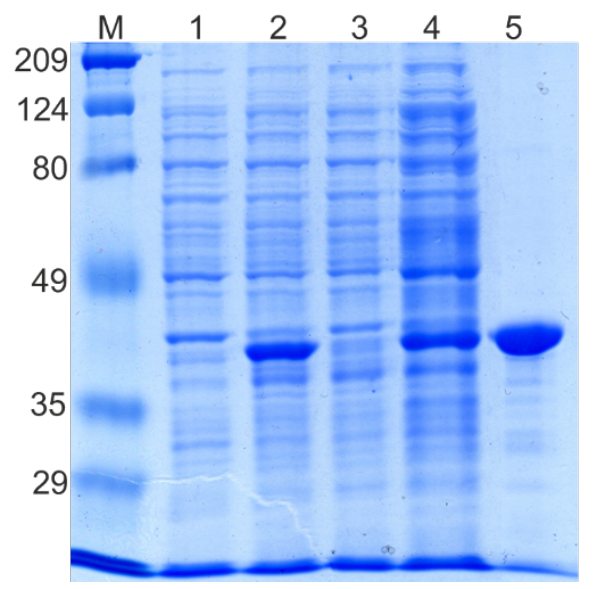

(b)

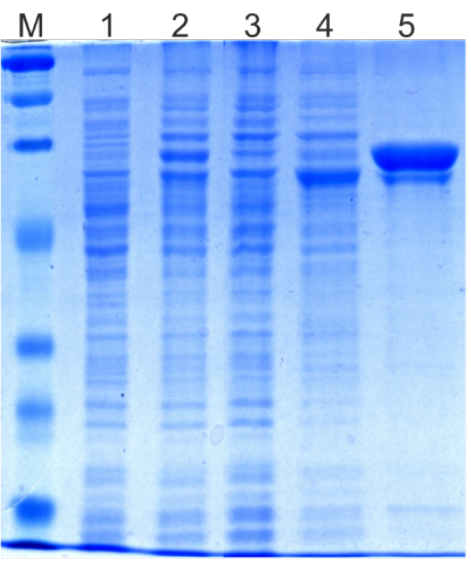

(c)

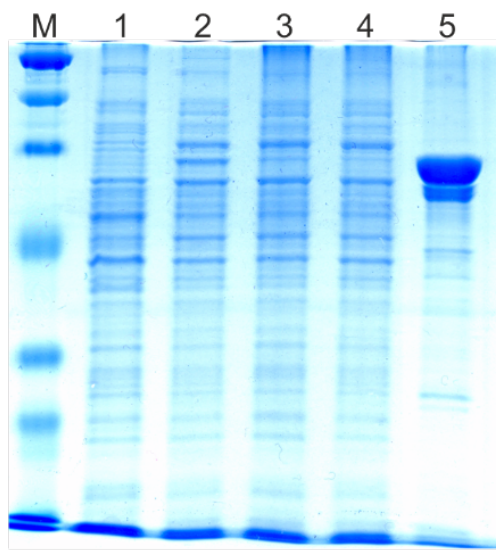

(e)

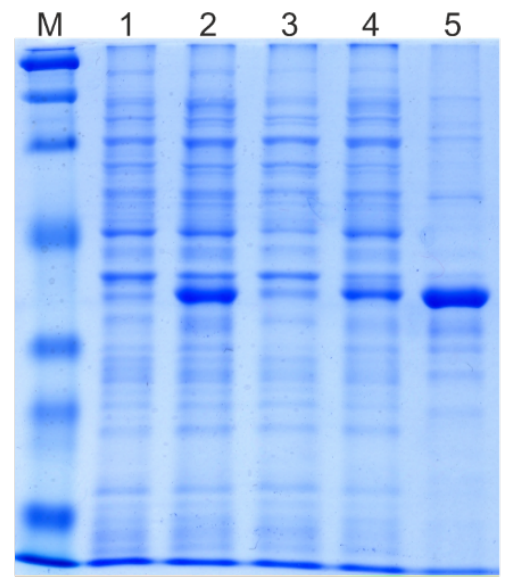

(a) BglC (theoretical Mw $=54.2 \mathrm{kDa}$ ), (b) BglC-CtDoc (theoretical Mw $=63.5 \mathrm{kDa}$ ), (c) BglCAcDoc (theoretical Mw $=63.9 \mathrm{kDa}$ ), (d) GFP-CtDoc (theoretical Mw $=36.5 \mathrm{kDa}$ ), and (e) CFP-AcDoc (theoretical $\mathrm{Mw}=37.3 \mathrm{kDa}$ ). Lane 1, cell-free extract prepared from sample collected before induction of gene expression; lane 2, cell-free extract prepared from sample collected at the end of the culture (after induction of gene expression); lane 3, flow-through fraction from NiNTA-based protein purification; lane 4, wash fraction from protein purification; lane 5, sample of purified protein obtained after merging elution fractions with the highest $\beta$-glucosidase activity (gels $a, b$, and $c$ ) or elution fractions with the highest fluorescence (gels d and e). Defined quantity of total protein $(5 \mu \mathrm{g})$ was loaded in each well. $\mathrm{M}$, protein marker (values are in $\mathrm{kDa}$ ). 
Supplementary Figure S4. Effect of an autotransporter expression on viability of the host bacterium Pseudomonas putida EM42.

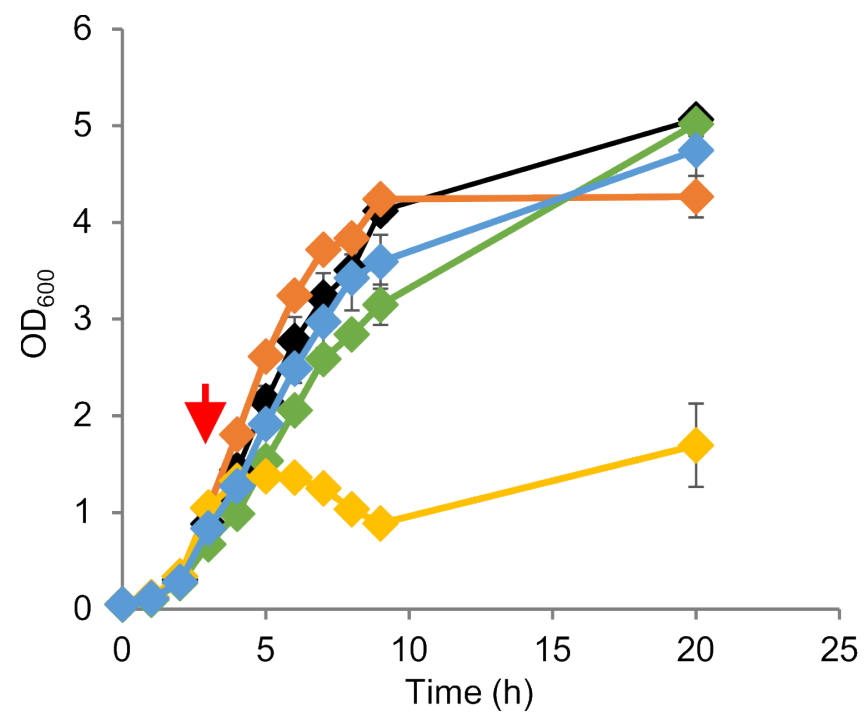

Cells were cultured in $20 \mathrm{~mL}$ of lysogeny broth at $30{ }^{\circ} \mathrm{C}$ with shaking $(170 \mathrm{rpm})$. Expression of an autotransporter gene (est $P$ in yellow, int in green, igA in blue and $a g 43$ in orange) cloned in the pSEVA238 plasmid (control with empty pSEVA238 is shown in black) was induced $3 \mathrm{~h}$ after culture start (red arrow) with $1 \mathrm{mM} 3$-methylbenzoate. Data points show mean values \pm SD from two biological replicates. 
Supplementary Figure S5. Expression and display analysis of three different autotransporters with the CtCoh passenger in Pseudomonas putida EM42 and EM371.

(a)

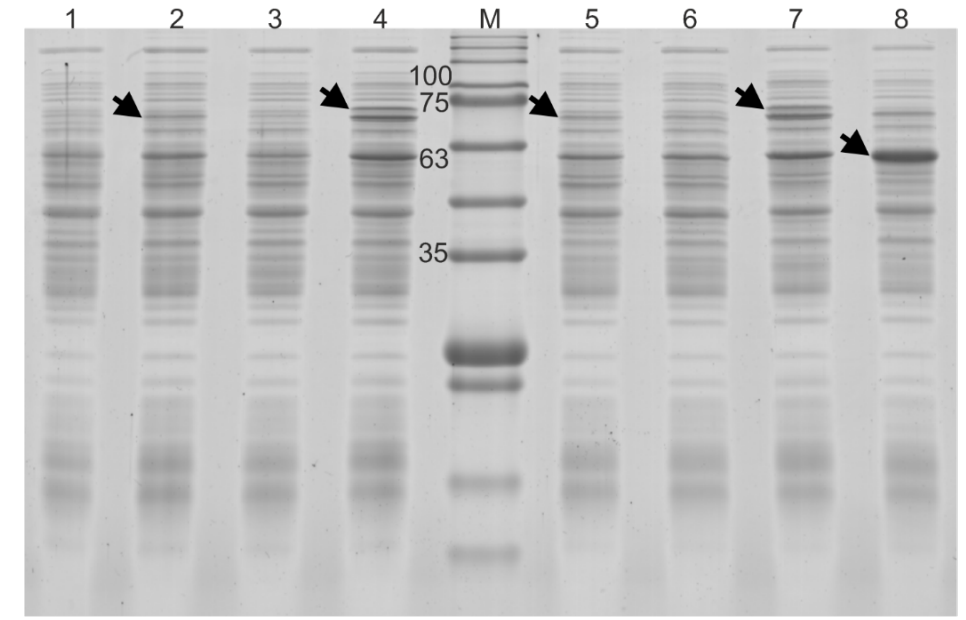

(b)

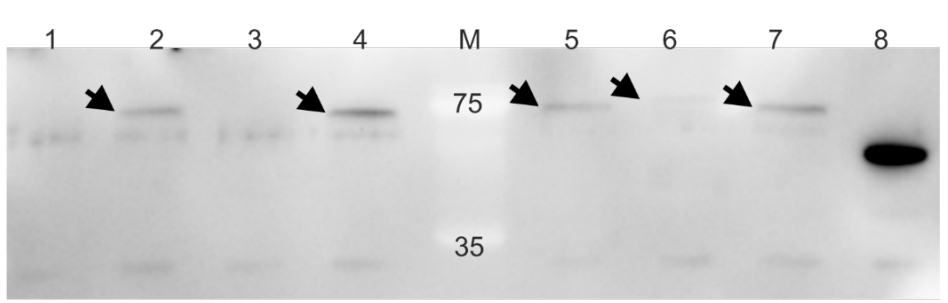

(c)

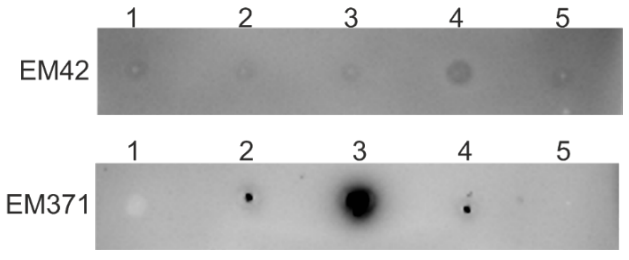

(a) Sodium dodecyl sulfate polyacrylamide gel electrophoresis (12\% gel) and (b) parallel western blot analysis of cell lysates obtained from P. putida EM42 pSEVA238 (1, negative control), P. putida EM42 pSEVA238_igAAT-ctCoh (2), P. putida EM42 pSEVA238_intATctCoh (3), P. putida EM42 pSEVA238b_ag43At-ctCoh (4), P. putida EM371 pSEVA238_igAAT-ctCoh (5), P. putida EM371 pSEVA238_intAT-ctCoh (6), P. putida EM371 pSEVA238b_ag43At-ctCoh (7), and P. putida EM42 pSEVA238b_bglC (8, positive control) grown in lysogeny broth and induced for $4.5 \mathrm{~h}$ with $0.5 \mathrm{mM} 3$-methylbenzoate. Theoretical molecular weights of detected proteins whose bands are designated with arrows are $67.7 \mathrm{kDa}$ for IgAAT-CtCoh, $87.6 \mathrm{kDa}$ for IntAT-CtCoh, $69.6 \mathrm{kDa}$ for Ag43AT-CtCoh, and $53.4 \mathrm{kDa}$ for BglC control. Equal amounts of total protein $(5 \mu \mathrm{g})$ were loaded per gel well. $\mathrm{M}$ is protein marker, values are in $\mathrm{kDa}$. (c) Dot blot analysis of whole pre-induced P. putida EM42 and EM371 cells expressing igAAT-ctCoh (1), intAT-ctCoh (2), ag43AT-ctCoh (3), bglC (4, cytoplasmic protein control), or bearing empty pSEVA238 plasmid (5, negative control). In case of (b) and (c), proteins with 6xHis tag were detected using mouse anti-His tag monoclonal antibody-HRP conjugate (Thermo Fisher Scientific, USA). 
Supplementary Figure S6. Expression analysis of three scaffoldin variants displayed via Ag43 autotransporter in Pseudomonas putida EM42 and EM371.

(a)

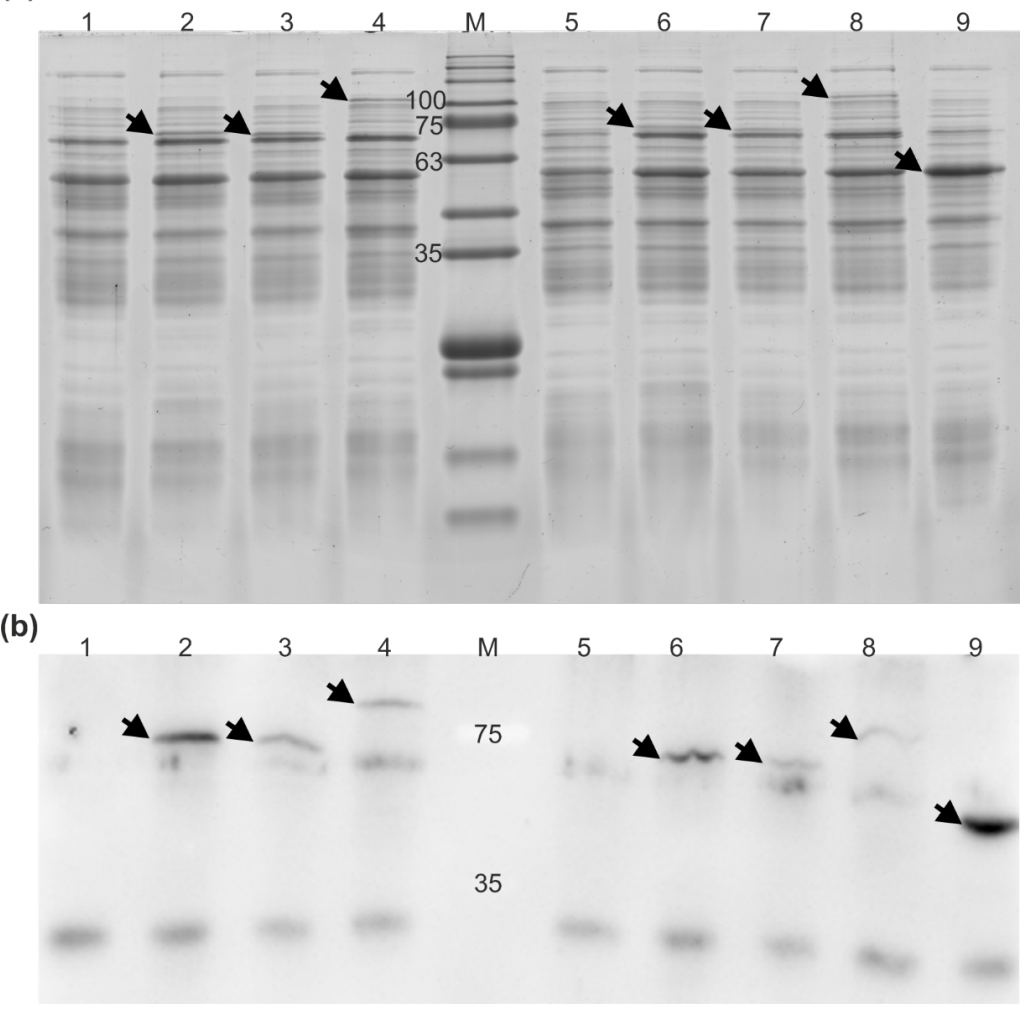

(a) Sodium dodecyl sulfate polyacrylamide gel electrophoresis (12\% gel) and (b) parallel western blot analysis of cell lysates obtained from $P$. putida EM42 pSEVA238b_ag43AT (1, negative control), $P$. putida EM42 pSEVA238b_ag43AT-acCoh (2), P. putida EM42 pSEVA238b_ag43AT-ctCoh (3), P. putida EM42 pSEVA238b_ag43At-acCoh-ctCoh (4), P. putida EM371 pSEVA238b_ag43AT (5, negative control), $P$. putida EM371 pSEVA238b_ag43AT-acCoh (6), P. putida EM371 pSEVA238b_ag43At-ctCoh (7), P. putida EM371 pSEVA238b_ag43At-acCoh-ctCoh (8), and P. putida EM42 pSEVA238b_bglC (8, positive control) grown in lysogeny broth and induced for $4.5 \mathrm{~h}$ with $0.5 \mathrm{mM} 3$ methylbenzoate. Theoretical molecular weights of detected proteins whose bands are indicated by arrows are $69.9 \mathrm{kDa}$ for Ag43AT-AcCoh, $69.6 \mathrm{kDa}$ for Ag43AT-CtCoh, 88.1 kDa for $\mathrm{Ag} 43 \mathrm{AT}-\mathrm{AcC}$ Ch-CtCoh, and $53.4 \mathrm{kDa}$ for BglC control. Equal amounts of total protein - $5 \mu \mathrm{g}$ in (a) and $10 \mu \mathrm{g}$ in (b) - were loaded per gel well. $\mathrm{M}$ is protein marker, values are in $\mathrm{kDa}$. In (b), proteins with 6xHis tag were detected using mouse anti-His tag monoclonal antibody-HRP conjugate (Thermo Fisher Scientific, USA). 


\section{Supplementary sequences}

\section{scaf19LKT}

ATGGCAAACACCCCTGTCAGCGGTAACCTCAAGGTCGAGTTCTACAACAGCAACCCGAGCGACACGACGAACAGC ATCAACCCGCAGTTCAAGGTCACGAACACCGGCAGCAGCGCAATCGACCTCTCCAAGCTGACTCTCCGTTACTAT TATACCGTCGATGGCCAGAAGGACCAGACCTTCTGGTGCGACCACGCTGCAATCATCGGCAGCAACGGTAGCTAC AACGGCATCACCTCCAACGTCAAGGGCACCTTCGTCAAGATGTCCTCCTCGACCAACAACGCAGACACCTACCTG GAGATCAGCAGCACCGGCGGTACGCTGGAGCCTGGTGCACACGTTCAAATCCAGGGTCGTTTCGCAAAGAACGAC TGGAGCAACTACACCCAGTCGAACGACTACTCGTTCAAGAGCGCCTCGCAGTTCGTCGAGTGGGACCAGGTCACC GCATACCTGAACGGCGTCCTGGTCTGGGGTAAGGAGCCTGGTGGTAGCGTTGTTCCATCGACTCAGCCAGTTACC ACCCCACCTGCTACTACCAAGCCTCCTGCTACCACTAAGCCACCAGCTACCACTATCCCGCCATCGGGTTCCGAT TTGCAGGTGGACATCGGTAGCACTAGCGGTAAGGCTGGTAGCGTAGTGAGCGTTCCAATCACCTTCACCAACGTG CCGAAGTCGGGCATCTACGCGCTGAGCTTCCGGACTAACTTCGACCCCCAGAAGGTTACCGTGGCTTCCATCGAC GCCGGCTCGTTGATTGAGAACGCCAGCGATTTCACTACCTACTACAACAACGAGAACGGCTTCGCCTCGATGACC TTCGAGGCCCCGGTGGACCGGGCCCGCATTATTGACAGCGATGGCGTATTCGCCACTATCAACTTCAAGGTGAGC GACAGCGCCAAGGTGGGCGAACTGTACAACATCACCACCAACAGCGCCTACACCTCGTTCTACTACTCCGGGACC GACGAGATCAAGAACGTGGTGTACAACGACGGCAAGATCGAGGTGATCGCCAGCCCGACCCCGACTCAGTCGGCC ACCCCGACGGTAACCCCGTCGGCCACGGCCACGCCGACGCAATCCGCCACGCCGACCGTAACGCCCTCCGATGGC GTAGTAGTGGAAATTGGCAAAGTGACGGGCAGCGTGGGCACCACCGTGGAAATTCCGGTGTACTTTCGCGGCGTG CCCTCCAAAGGCATTGCCAATTGTGATTTTGTGTTCGCTACGATCCGAATGTGTTGGAAATTATTGGGATTGAT CCCGGCGATATCATCGTGGACCCGAACCCGACCAAGAGCTTTGATACCGCCATCTACCCGGATCGCAAGATCATC GTGTTCCTGTTCGCGGAAGACAGCGGCACCGGCGCGTATGCCATTACCAAGGATGGCGTGTTCGCCAAAATCCGC GCCACCGTGAAATCGTCCGCGCCGGGCTATATTACCTTTGACGAAGTGGGCGGCTTTGCCGATAATGACTTGGTG GAACAGAAGGTCTCGTTCATCGACGGGGGGGTGAACGTGGGCAACGCCACCCCGACCAAAGGCGCCACCCCCACC AATACCGCGACGCCGACCAAATCGGCGACGGCGACGCCCACCCGCCCCTCGGCCCCGACGAATACGCCGACCAAT ACCCCGTCCTCGCCCGGCAATAAAATGAAAATTCAAATCGGGGACGTCAAAGCGAATCAAGGCGACACCGTGATC GTCCCGATCACCTTTAATGAAGTGCCGGTCATGGGGGTGAATAATTGCAACTTCACCCTGGCGTATGACAAAAAC ATCATGGAGTTCATCAGCGCGGACGCCGGGGACATCGTCACCCTGCCCATGGCGAACTATAGCTATAACATGCCC AGCGACGGGCTGGTCAAATTCCTGTATAACGACCAAGCGCAGGGCGCCATGTCGATCAAAGAAGACGGCACCTTC GCGAACGTGAAGTTCAAGATCAAGCAGTCCGCCGCCTTCGGGAAGTACTCGGTCGGCATCAAGGCCATCGGCTCG ATCTCCGCGCTGAGCAACTCCAAGCTGATCCCCATCGAATCGATCTTCAAGGACGGCAGCATCACCGTCACCAAC CTGGAACACCATCACCATCATCACTAA

\section{bglC-ctDoc}

ATGCACCATCACCATCACCATACCTCGCAATCGACGACTCCTCTGGGCAATCTCGAGGAGACTCCCAAACCGGAT ATCCGCTTCCCGTCCGATTTCGTGTGGGGAGTGGCGACCGCTTCGTTCCAGATCGAAGGCTCCACCACGGCCGAC GGCCGCGGCCCCAGCATCTGGGACACCTTCTGCGCCACTCCGGGCAAGGTCGAGAACGGCGACACGGGCGACCCT GCCTGCGACCACTACAACCGGTACCGCGATGACGTGGCCTTGATGCGGGAGCTGGGCGTGGGCGCCTACCGCTTC TCCATCGCCTGGCCGCGGATCCAGCCCGAGGGCAAGGGCACGCCCGTGGAGGCCGGGCTGGACTTCTACGACCGG CTTGTGGACTGCCTGCTGGAGGCCGGCATCGAGCCGTGGCCGACCCTCTACCACTGGGACCTGCCGCAGGCGCTG GAGGACGCGGGCGGCTGGCCCAACCGGGACACGGCCAAGCGGTTCGCCGACTACGCGGAGATCGTCTACCGCCGG CTCGGCGACCGGATCACCAACTGGAACACGCTCAACGAGCCGTGGTGCTCCGCGTTCCTGGGCTACGCCTCCGGC GTGCACGCCCCGGGCCGCCAGGAGCCGGCTGCTGCGCTGGCCGCCGCCCACCACCTGATGCTGGGCCACGGGCTG GCCGCTGCCGTGATGCGGGACTTGGCGGGCCAGGCCGGACGTTCCGTGCGGATCGGTGTCGCGCACAACCAGACC ACGGTCCGTCCCTACACTGACAGTGAGGCCGACCGGGACGCTGCGCGCCGGATTGACGCCCTGCGGAACCGCATC TTCACCGAGCCGCTGGTGAAGGGCCGCTACCCGGAGGACCTGATCGAGGACGTCGCCGCGGTCACCGACTACAGC TTCGTCCAGGACGGCGACCTGAAGACCATCTCCGCCAACCTGGACATGATGGGCGTCAACTTCTACAACCCGAGC TGGGTGTCAGGCAACCGGGAGAACGGGGGCTCCGACCGGCTGCCCGACGAGGGCTACTCGCCGTCGGTCGGCAGC GAGCATGTCGTGGAGGTGGACCCCGGCCTGCCGGTGACCGCCATGGGCTGGCCGATCGACCCGACCGGGCTGTAC GACACGCTGACCCGGCTGGCCAACGACTACCCGGGCCTGCCGCTGTACATCACCGAGAACGGCGCCGCCTTCGAG GACAAGGTGGTCGACGGCGCGGTGCACGACACCGAGCGGATCGCCTACCTGGACTCGCACCTGCGGGCCGCGCAC GCTGCCATTGAGGCGGGCGTGCCGCTCAAGGGCTACTTCGCCTGGTCGTTCATGGACAACTTCGAGTGGGCCCTC GGGTACGGGAAGCGGTTCGGCATCGTGCACGTGGACTACGAGAGCCAGACGCGCACGGTGAAGGACAGCGGCTGG TGGTACTCCCGGGTGATGCGCAACGGGGGAATCTTCGGACAGGAATCCGGGGGTGGTTCCGCTAGCGGGACGCCC AGCACCAAACTGTACGGCGATGTCAATGATGACGGCAAAGTGAACTCGACCGACGCCGTCGCCCTGAAGCGGTAT GTGCTGCGGTCGGGCATCAGCATCAACACCGACAATGCCGACCTGAATGAAGACGGCCGGGTCAATTCGACCGAC CTGGGCATTCTGAAGCGCTATATTCTCAAAGAAATTGACACGCTGCCGTACAAGAACCACCACCATCACCACCAC TAA 


\section{bglC-acDoc}

ATGCACCATCACCATCACCATACCTCGCAATCGACGACTCCTCTGGGCAATCTCGAGGAGACTCCCAAACCGGAT ATCCGCTTCCCGTCCGATTTCGTGTGGGGAGTGGCGACCGCTTCGTTCCAGATCGAAGGCTCCACCACGGCCGAC GGCCGCGGCCCCAGCATCTGGGACACCTTCTGCGCCACTCCGGGCAAGGTCGAGAACGGCGACACGGGCGACCCT GCCTGCGACCACTACAACCGGTACCGCGATGACGTGGCCTTGATGCGGGAGCTGGGCGTGGGCGCCTACCGCTTC TCCATCGCCTGGCCGCGGATCCAGCCCGAGGGCAAGGGCACGCCCGTGGAGGCCGGGCTGGACTTCTACGACCGG CTTGTGGACTGCCTGCTGGAGGCCGGCATCGAGCCGTGGCCGACCCTCTACCACTGGGACCTGCCGCAGGCGCTG GAGGACGCGGGCGGCTGGCCCAACCGGGACACGGCCAAGCGGTTCGCCGACTACGCGGAGATCGTCTACCGCCGG CTCGGCGACCGGATCACCAACTGGAACACGCTCAACGAGCCGTGGTGCTCCGCGTTCCTGGGCTACGCCTCCGGC GTGCACGCCCCGGGCCGCCAGGAGCCGGCTGCTGCGCTGGCCGCCGCCCACCACCTGATGCTGGGCCACGGGCTG GCCGCTGCCGTGATGCGGGACTTGGCGGGCCAGGCCGGACGTTCCGTGCGGATCGGTGTCGCGCACAACCAGACC ACGGTCCGTCCCTACACTGACAGTGAGGCCGACCGGGACGCTGCGCGCCGGATTGACGCCCTGCGGAACCGCATC TTCACCGAGCCGCTGGTGAAGGGCCGCTACCCGGAGGACCTGATCGAGGACGTCGCCGCGGTCACCGACTACAGC TTCGTCCAGGACGGCGACCTGAAGACCATCTCCGCCAACCTGGACATGATGGGCGTCAACTTCTACAACCCGAGC TGGGTGTCAGGCAACCGGGAGAACGGGGGCTCCGACCGGCTGCCCGACGAGGGCTACTCGCCGTCGGTCGGCAGC GAGCATGTCGTGGAGGTGGACCCCGGCCTGCCGGTGACCGCCATGGGCTGGCCGATCGACCCGACCGGGCTGTAC GACACGCTGACCCGGCTGGCCAACGACTACCCGGGCCTGCCGCTGTACATCACCGAGAACGGCGCCGCCTTCGAG GACAAGGTGGTCGACGGCGCGGTGCACGACACCGAGCGGATCGCCTACCTGGACTCGCACCTGCGGGCCGCGCAC GCTGCCATTGAGGCGGGCGTGCCGCTCAAGGGCTACTTCGCCTGGTCGTTCATGGACAACTTCGAGTGGGCCCTC GGGTACGGGAAGCGGTTCGGCATCGTGCACGTGGACTACGAGAGCCAGACGCGCACGGTGAAGGACAGCGGCTGG TGGTACTCCCGGGTGATGCGCAACGGGGGAATCTTCGGACAGGAATCCGGGGGTGGTTCCGCTAGCAAGTTCATC TACGGCGACGTGGACGGCAACGGCAGCGTCCGCATCAACGACGCTGTGCTGATCCGTGACTACGTTCTGGGCAAG ATCAACGAGTTCCCCTACGAATATGGTATGTTAGCTGCTGATGTAGATGGTAACGGCTCCATCAAGATCAACGAC GCCGTCCTGGTGCGCGACTACGTGCTGGGCAAGATCTTCCTGTTCCCCGTCGAAGAAAAGGAAGAACACCACCAT CACCATCACTAA

\section{gfp-ctDoc}

ATGAGTAAAGGAGAAGAACTTTTCACCGGTGTTGTTCCGATCCTGGTTGAACTGGATGGTGATGTTAACGGCCAC AAATTCTCTGTTCGTGGTGAAGGTGAAGGTGATGCAACCAACGGTAAACTGACCCTGAAATTCATCTGCACTACC GGTAAACTGCCGGTTCCATGGCCGACTCTGGTGACTACCCTGACCTATGGTGTTCAGTGTTTTTCTCGTTACCCG GATCACATGAAGCAGCATGATTTCTTCAAATCTGCAATGCCGGAAGGTTATGTACAGGAGCGCACCATTTCTTTC AAAGACGATGGCACCTACAAAACCCGTGCAGAGGTTAAATTTGAAGGTGATACTCTGGTGAACCGTATTGAACTG AAAGGCATTGATTTCAAAGAGGACGGCAACATCCTGGGCCACAAACTGGAATATAACTTCAACTCCCATAACGTT TACATCACCGCAGACAAACAGAAGAACGGTATCAAAGCTAACTTCAAAATTCGCCATAACGTTGAAGACGGTAGC GTACAGCTGGCGGACCACTACCAGCAGAACACTCCGATCGGTGATGGTCCGGTTCTGCTGCCGGATAACCACTAC CTGTCCACCCAGTCTAAACTGTCCAAAGACCCGAACGAAAAGCGCGACCACATGGTGCTGCTGGAGTTCGTTACT GCCGCAGGTATCACGCACGGCATGGATGAACTATACAAAGAATTCGAGCTCTCCGGGGGTGGTTCCGCTAGCGGG ACGCCCAGCACCAAACTGTACGGCGATGTCAATGATGACGGCAAAGTGAACTCGACCGACGCCGTCGCCCTGAAG CGGTATGTGCTGCGGTCGGGCATCAGCATCAACACCGACAATGCCGACCTGAATGAAGACGGCCGGGTCAATTCG ACCGACCTGGGCATTCTGAAGCGCTATATTCTCAAAGAAATTGACACGCTGCCGTACAAGAACCACCACCATCAC CACCACTAA

\section{cfp-acDoc}

ATGGGCCATCATCATCACCATCACGTGAGCAAGGGCGAGGAGCTGTTCACCGGGGTGGTGCCCATCCTGGTCGAG CTGGACGGCGACGTAAACGGCCACAAGTTCAGCGTGTCCGGCGAGGGCGAGGGCGATGCCACCTACGGCAAGCTG ACCCTGAAGTTCATCTGCACCACCGGCAAGCTGCCCGTGCCCTGGCCCACCCTCGTGACCACCCTGACCTGGGGC GTGCAGTGCTTCGCCCGCTACCCCGACCACATGAAGCAGCACGACTTCTTCAAGTCCGCCATGCCCGAAGGCTAC GTCCAGGAGCGCACCATCTTCTTCAAGGACGACGGCAACTACAAGACCCGCGCCGAGGTGAAGTTCGAGGGCGAC ACCCTGGTGAACCGCATCGAGCTGAAGGGCATCGACTTCAAGGAGGACGGCAACATCCTGGGGCACAAGCTGGAG TACAACGCCATCAGCGACAACGTCTATATCACCGCCGACAAGCAGAAGAACGGCATCAAGGCCAACTTCAAGATC CGCCACAACATCGAGGACGGCAGCGTGCAGCTCGCCGACCACTACCAGCAGAACACCCCCATCGGCGACGGCCCC GTGCTGCTGCCCGACAACCACTACCTGAGCACCCAGTCCAAGCTGAGCAAAGACCCCAACGAGAAGCGCGATCAC ATGGTCCTGCTGGAGTTCGTGACCGCCGCCGGGATCACTCTCGGCATGGACGAGCTGTACAAGGGTACCAAATTT AтATATGGTGATGTTGATGGTAATGGAAGTGTAAGAATTAATGATGCTGTCCTAATAAGAGACTATGTATTAGGA AAAATCAATGAATTCCCATATGAATATGGTATGCTTGCAGCAGATGTTGATGGTAATGGAAGTATAAAAATTAAT GATGCTGTTCTAGTAAGAGACTACGTGTTAgGAAAGATATTTTTATTCCCTGTTGAAGAGAAAGAAGAACTCGAG CACCACCACCACCACCACTGA 


\section{ig $\boldsymbol{A} A \boldsymbol{T}$}

ATGAAATACCTATTGCCTACGGCAGCCGCTGGATTGTTATTACTCGCCGCCCAGCCGGCCATGGCGATCGTTCTT GGTGCGCCGGTGCCGTATCCCGATCCGCTGGAACCGCGTGCCGCCGCCGCGGCCGCGAATTCGAGCTCGGTACCC GGGGATCCTCTAGAGTCGACCTGCAGGCATGCAAGCTTGCGGCCGCTCGACAATTCAGCCGCAATTAGTATGGCA AATCCACGTCCACCAACACCGCGGGCTGCTGCGGCCGTATTTTCATTGGATGATTATGATGCAAAAGACAATAGT GAATCATCAATAGGTAATTTAGCTCGTGTAATACCTAGAATGGGAAGGGAGCTAATTAATGATTATGAAGAAATC CCCTTGGAGGAGTTGGAAGATGAAGCGGAAGAAGAACGTCGCCAAGCAACGCAATTCCAACCCAAAAGTCGTAAC CGTAGAGCTATATCATCGGAACCATCATCTGATGAAGATGCATCTGAATCGGTTTCCACATCAGACAAACACCCT CAAGATAATACGGAACTTCATGAAAAAGTTGAGACGGCGGGTTTACAACCAAGAGCCGCGCAGCCGCGAACCCAA GCCGCCGCGCAAGCCGATGCAGTCAGCACCAATACTAACTCGGCTTTATCTGACGCAATGGCAAGCACGCAATCT ATCTTGTTGGATACAGGTGCTTCATTAACACGGCACATTGCACAAAAATCACGCGCTGATGCCGAAAAAAACAGT GTTTGGATGTCAAACACCGGTTATGGCCGTGATTATGCTTCCGCACAATATCGCCGGTTTAGTTCGAAACGCACG CAAACACAAATCGGCATTGACCGCAGCTTGTCCGAAAATATGCAGATAGGCGGAGTATTGACTTACTCTGACAGT CAGCATTCTTTTGATCTGGCGGGCGGCAAAAATACTTTTGTGCAAGCCAACCTTTATGGTAAGTATTATCTAAAT GATGCTTGGTATGTGGCCGGCGATATTGGTGCGGGCAGCTTGAGAAGCCGGTTACAAACGCAGCAAAAAGCAAAC TTTAACCGAACAAGCATCCAAACCGGCCTTACTTTGGGCAATACGCTGAAAATCAATCAATTCGAGATTGTCCCT AGTGCGGGTATCCGTTACAGCCGCCTGTCATCTGCTGATTACAAGTTGGGTGACGACAGTGTTAAAGTAAGTTCT ATGGCAGTGAAAACACTAACGGCCGGACTGGATTTTGCTTATCGGTTTAAAGTCGGCAACCTTACCGTAAAACCC TTGTTATCTGCTGCTTACTTTGCCAATTATGGCAAAGGCGGCGTGAATGTGGGCGGTAAATCCTTCGCCTATAAA GCAGATAATCAACAGCAATATTCAGCAGGCGCCGCGTTACTGTACCGTAATGTTACATTAAACGTAAATGGCAGT ATTACAAAAGGAAAACAATTGGAAAAACAAAAATCCGGACAAATTAAAATACAGATTCGTTTCTAA

\section{intAT}

ATGATTACTCATGGTTGTTATACCCGGACCCGGCACAAGCATAAGCTAAAAAAAACATTGATTATGCTTAGTGCT GGTTTAGGATTGTTTTTTTATGTTAATCAGAACTCATTTGCAAATGGTGAAAATTATTTTAAATTGGGTTCGGAT TCAAAACTGTTAACTCATGATAGCTATCAGAATCGCCTTTTTTATACGTTGAAAACTGGTGAAACTGTTGCCGAT CTTTCTAAATCGCAAGATATTAATTTATCGACGATTTGGTCGTTGAATAAGCATTTATACAGTTCTGAAAGCGAA ATGATGAAGGCCGCGCCTGGTCAGCAGATCATTTTGCCACTCAAAAAACTTCCCTTTGAATACAGTGCACTACCA CTTTTAGGTTCGGCACCTCTTGTTGCTGCTGGTGGTGTTGCTGGTCACACGAATAAACTGACTAAAATGTCCCCG GACGTGACCAAAAGCAACATGACCGATGACAAGGCATTAAATTATGCGGCACAACAGGCGGCGAGTCTCGGTAGC CAGCTTCAGTCGCGATCTCTGAACGGCGATTACGCGAAAGATACCGCTCTTGGTATCGCTGGTAACCAGGCTTCG TCACAGTTGCAGGCCTGGTTACAACATTATGGAACGGCAGAGGTTAATCTCCAGAGTGGTAATAACTTTGACGGT AGTTCACTGGACTTCTTATTACCGTTCTATGATTCCGAAAAAATGCTGGCATTTGGTCAGGTCGGAGCGCGTTAC ATTGACTCCCGCTTTACGGCAAATTTAGGTGCGGGTCAGCGTTTTTTCCTTCCTGCAAACATGTTGGGCTATAAC GTCTTCATTGATCAGGATTTTTCTGGTGATAATACCCGTTTAGGTATTGGTGGCGAATACTGGCGAGACTATTTC AAAAGTAGCGTTAACGGCTATTTCCGCATGAGCGGCTGGCATGAGTCATACAATAAGAAAGACTATGATGAGCGC CCAGCAAATGGCTTCGATATCCGTTTTAATGGCTATCTACCGTCATATCCGGCATTAGGCGCCAAGCTGATATAT GAGCAGTATTATGGTGATAATGTTGCTTTGTTTAATTCTGATAAGCTCCAGTCGAATCCTGGTGCGGCGACCGTT GGTGTAAACTATACTCCGATTCCTCTGGTGACGATGGGGATCGATTACCGTCATGGTACGGGTAATGAAAATGAT CTCCTTTACTCAATGCAGTTCCGTTATCAGTTTGATAAATCGTGGTCTCAGCAAATTGAACCACAGTATGTTAAC GAGTTAAGAACATTATCAGGCAGCCGTTACGATCTGGTTCAGCGTAATAACAATATTATTCTGGAGTACAAGAAG CAGGATATTCTTTCTCTGAATATTCCGCATGATATTAATGGTACTGAACACAGTACGCAGAAGATTCAGTTGATC GTTAAGAGCAAATACGGTCTGGATCGTATCGTCTGGGATGATAGTGCATTACGCAGTCAGGGCGGTCAGATTCAG CATAGCGGAAGCCAAAGCGCACAAGACTACCAGGCTATTTTGCCTGCTTATGTGCAAGGTGGCAGCAATATTTAT AAAGTGACGGCTCGCGCCTATGACCGTAATGGCAATAGCTCTAACAATGTACAGCTTACTATTACCGTTCTGTCG AATGGTCAAGTTGTTGACCAGGTTGGGGTAACGGACTTTACGGCGGATAAGACTTCGGCTAAAGCGGATAACGCC GATACCATTACTTATACCGCGACGGTGAAAAAGAATGGGGTAGCTCAGGCTAATGTCCCTGTTTCATTTAATATT GTTTCAGGAACTGCAACTCTTGGGGCAAATAGTGCCAAAACGGATGCTAACGGTAAGGCAACCGTAACGTTGAAG TCGAGTACGCCAGGACAGGTCGTCGTGTCTGCTAAAACCGCGGAGATGACTTCAGCACTTAATGCCAGTGCGGTT ATATTTTTGATGCGGCCGCGAATTCGAGCTCGGTACCCGGGGATCCTCTAGAGTCGACCTGCAGGCATGCAAGC TTGCGGCCGCCCGGTGCGCCGGTGCCGTATCCCGATCCGCTGGAACCGCGTTAA 


\section{$\operatorname{ag} 43 A T$}

ATGAAACGACATCTGAATACCTGCTACAGGCTGGTATGGAATCACATGACGGGCGCTTTCGTGGTTGCCTCCGAA CTGGCCCGCGCACGGGGTAAACGTGGCGGTGTGGCGGTTGCACCGTCTCTTGCCGCAGTCACGTCACTCCCGGTG CTGGCTGCTGACAGATCTGCCATGGCGGAATTCGAGCTCGGTACCCTCGAGCTGAACGGATCCATTGACCCCACG AATGTCACTCTCGCCTCCGGTGCCACCTGGAATATCCCCGATAACGCCACGGTGCAGTCGGTGGTGGATGACCTC AGCCATGCCGGACAGATTCATTTCACCTCCACCCGCACAGGGAAGTTCGTACCGGCAACCCTGAAAGTGAAAAAC CTGAACGGACAGAATGGCACCATCAGCCTGCGTGTACGCCCGGATATGGCACAGAACAATGCTGACAGACTGGTC ATTGACGGCGGCAGGGCAACCGGAAAAACCATCCTGAACCTGGTGAACGCCGGCAACAGTGCGTCGGGGCTGGCG ACCAGCGGTAAGGGTATTCAGGTGGTGGAAGCCATTAACGGTGCCACCACGGAGGAAGGGGCCTTTGTCCAGGGG AACAGGCTGCAGGCCGGTGCCTTTAACTACTCCCTCAACCGGGACAGTGATGAGAGCTGGTATCTGCGCAGTGAA AATGCTTATCGTGCAGAAGTCCCCCTGTATGCCTCCGTGCTGACACAGGCAATGGACTATGACCGGATTGTGGCA GGCTCCCGCAGCCATCAGACCGGTGTAAATGGTGAAAACAACAGCGTCCGTCTCAGCATTCAGGGCGGTCATCTC GGTCACGATAACAATGGCGGTATTGCCCGTGGGGCCACGCCGGAAAGCAGCGGCAGCTATGGATTCGTCCGTCTG GAGGGTGACCTGATGAGAACAGAGGTTGCCGGTATGTCTGTGACCGCGGGGGTATATGGTGCTGCTGGCCATTCT TCCGTTGATGTTAAGGATGATGACGGCTCCCGTGCCGGCACGGTCCGGGATGATGCCGGCAGCCTGGGCGGATAC CTGAATCTGGTACACACGTCCTCCGGCCTGTGGGCTGACATTGTGGCACAGGGAACCCGCCACAGCATGAAAGCG TCATCGGACAATAACGACTTCCGCGCCCGGGGCTGGGGCTGGCTGGGCTCACTGGAAACCGGTCTGCCCTTCAGT ATCACTGACAACCTGATGCTGGAGCCACAACTGCAGTATACCTGGCAGGGACTTTCCCTGGATGACGGTAAGGAC AACGCCGGTTATGTGAAGTTCGGGCATGGCAGTGCACAGCATGTGCGTGCCGGTTTCCGTCTGGGCAGCCACAAC GATATGACCTTTGGCGAAGGCACCTCATCCCGTGCCCCCCTGCGTGACAGTGCAAAACACAGTGTGAGTGAATTA CCGGTGAACTGGTGGGTACAGCCTTCTGTTATCCGCACCTTCAGCTCCCGGGGAGATATGCGTGTGGGGACTTCC ACTGCAGGCAGCGGGATGACGTTCTCTCCCTCACAGAATGGCACATCACTGGACCTGCAGGCCGGACTGGAAGCC CGTGTCCGGGAAAATATCACCCTGGGCGTTCAGGCCGGTTATGCCCACAGCGTCAGCGGCAGCAGCGCTGAAGGG TATAACGGTCAGGCCACACTGAATGTGACCTTCTGA

\section{estPAT}

ATGCGAAAAGCCCCGTTATTGCGCTTTACCCTCGCTTCACTGGCCCTGGCCTGTAGCCAGGCGTTGGCCGGTGCG CCGGTGCCGTATCCGGACCCGCTGGAACCGCGTGGATCCGTCGACTCTAGACTGCAGTCGGTCCACCCGACCATC GCGGGTCAGCAGCTGATTGCCGATTACGCCTACTCGATCCTCGCGGCCCCCTGGGAACTGACCCTGCTACCGGAA ATGGCCCACGCCAGCCTGCGGGCTCACCAGGATGAGTTGCGTAATCAGTGGCAGACGCCTTGGCAAGCAGTTGGC CAATGGCAAGCCTTTGTCGCCAGCGGCGCTCAGGACCTGGACTTCGACGGCCAGCACAGCGCGGCCAGCGGTGAC GGCCGCGGCTACAACCTGACCGTGGGCGGCAGCTATCGCCTGAACGACGCCTGGCGCCTGGGCCTGGCCGGCGGT GCAAACCGGCAGAAGCTGGAAGCTGGTGAACAGGACTCGGACTACAAGCTGAACAGTTATATGGCCAGTGCCTTT GCCCAATACCGCCAGGACCGCTGGTGGGCCGACGCGGCGCTGACCGCCGGGCACCTGGATTACAGCGACCTCAAG CGTACCTTCGCCCTGGGCGTGAATGACCGCAGTGAGAAGGGCGACACCGACGGCGAGGCCTGGGCAATGTCCGGG CGGCTGGGCTACAACCTGGCGGCCGACACCAGCAACTGGCAGTTGGCACCTTTCATCAGTGCCGACTATGCGCGG GTGAAGGTGGATGGCTACGACGAGAAGAGCGGACGTTCGACGGCGCTTGGCTTCGATGACCAGGAGCGCACGTCA CGCCGCCTGGGCGTGGGGCTGCTGGGCAGTGTGCAGGTACTGCCAAGTACCCGGCTTTTCGCCGAGGTGGCGCAG GAGCATGAGTTCGAGGACGACGAGCAGGATGTGACGATGCACCTGACCAGCTTGCCGGCGAATGACTTCACCCTG ACCGGGTATACGCCGCACAGCGACCTGACCCGGGCGAGCCTGGGTGTGAGCCATGAACTGGTGGCAGGGGTGCAT TTGCGCGGGAACTACAACTGGCGCAAGAGTGATGAGTTGACGCAACAGGGTATTAGCGTGGGGGTTAGCGTGGAC TTCTGA 
(1) Grant, S. G., Jessee, J., Bloom, F. R., and Hanahan, D. (1990) Differential plasmid rescue from transgenic mouse DNAs into Escherichia coli methylation-restriction mutants. Proc. Natl. Acad. Sci. U. S. A. 87, 4645-4649.

(2) Manoil, C., and Beckwith, J. (1985) TnphoA: a transposon probe for protein export signals. Proc. Natl. Acad. Sci. U. S. A. 82, 8129-8133.

(3) Boyer, H. W., and Roulland-Dussoix, D. (1969) A complementation analysis of the restriction and modification of DNA in Escherichia coli. J. Mol. Biol. 41, 459-472.

(4) Martínez-García, E., Nikel, P. I., Aparicio, T., and de Lorenzo, V. (2014) Pseudomonas 2.0: genetic upgrading of $P$. putida KT2440 as an enhanced host for heterologous gene expression. Microb. Cell Factories 13, 159.

(5) Martinez-Garcia, E., Fraile, S., Rodriguez-Espeso, D., Vecchietti, D., Bertoni, G., and Lorenzo, V. de. (2020) The naked cell: emerging properties of a surfome-streamlined Pseudomonas putida strain. bioRxiv 2020.05.17.100628.

(6) Kessler, B., de Lorenzo, V., and Timmis, K. N. (1992) A general system to integrate lacZ fusions into the chromosomes of gram-negative eubacteria: regulation of the Pm promoter of the TOL plasmid studied with all controlling elements in monocopy. Mol. Gen. Genet. $M G G 233,293-301$.

(7) Silva-Rocha, R., Martínez-García, E., Calles, B., Chavarría, M., Arce-Rodríguez, A., de Las Heras, A., Páez-Espino, A. D., Durante-Rodríguez, G., Kim, J., Nikel, P. I., Platero, R., and de Lorenzo, V. (2013) The Standard European Vector Architecture (SEVA): a coherent platform for the analysis and deployment of complex prokaryotic phenotypes. Nucleic Acids Res. 41, D666-675.

(8) Dvořák, P., and de Lorenzo, V. (2018) Refactoring the upper sugar metabolism of Pseudomonas putida for co-utilization of cellobiose, xylose, and glucose. Metab. Eng. 48, 94-108.

(9) Vazana, Y., Barak, Y., Unger, T., Peleg, Y., Shamshoum, M., Ben-Yehezkel, T., Mazor, Y., Shapiro, E., Lamed, R., and Bayer, E. A. (2013) A synthetic biology approach for evaluating the functional contribution of designer cellulosome components to deconstruction of cellulosic substrates. Biotechnol. Biofuels 6, 182.

(10) Muñoz-Gutiérrez, I., Moss-Acosta, C., Trujillo-Martinez, B., Gosset, G., and Martinez, A. (2014) Ag43-mediated display of a thermostable $\beta$-glucosidase in Escherichia coli and its use for simultaneous saccharification and fermentation at high temperatures. Microb. Cell Factories 13, 106. 